\title{
Optimización de Órdenes de Producción Utilizando Algoritmo Genético
}

\author{
Production Order Optimization Using Genetic Algorithm
}

\author{
Victor Quezada-Aguilar ${ }^{a}$, José Carlos Quezada-Quezada ${ }^{a}$, Gaby Yolanda Vega-Cano ${ }^{a}$, \\ Francisco Quiroz-Aguilar ${ }^{b}$, Leopoldo Viveros-Rosas ${ }^{b}$, Ricardo Rodríguez-Figueroa ${ }^{b}$.
}

\begin{abstract}
:
Today, modern production systems require high standards for scheduling or sequencing production orders. Processes and procedures in industries require three basic elements for proper management: Planning, scheduling and production control. The article develops the optimization of a simple production system, with basic characteristics of the same system, which has 5 workstations where the processing sequence is linear. The problem is modeled with 6,10 and 13 production orders, each increase in the number of orders the allocation of production becomes more complex. The goal of the optimization system is to find the proper allocation that minimized the processing time of all production orders. As an optimization tool, the genetic algorithm is used due to the characteristics of the problem and the efficiency that has been demonstrated in optimization problems in most sciences. The number of solutions for the optimum of each problem raised decreases as the number of possible combinations of production orders increases, minimizing response times to the person in charge of making the decision on the allocation of production.
\end{abstract}

Keywords:

Production orders, Optimization, Genetic algorithm, Production system, Evolutionary algorithm

\section{Resumen:}

En la actualidad, los sistemas de producción modernos requieren de altos estándares de programación o secuenciación de las órdenes de producción. Los procesos y procedimientos en las industrias requieren de tres elementos básicos para una gestión adecuada: Planeación, programación y control de la producción. El artículo desarrolla la optimización de un sistema de producción simple, con características básicas propias del mismo sistema, el cual tiene 5 estaciones de trabajo donde la secuencia de procesamiento es lineal. El problema se modela con 6, 10 y 13 órdenes de producción, cada incremento en el número de órdenes la asignación de la producción se vuelve más compleja. El objetivo del sistema de optimización es encontrar la asignación adecuada que minimicé el tiempo de procesamiento de todas las órdenes de producción. Como herramienta de optimización se utiliza el algoritmo genético por las características del problema y por la eficiencia que a demostrado en problemas de optimización en la mayoría de las ciencias. El número de soluciones para el óptimo de cada problema planteado decrece con forme incrementa el número de combinaciones posibles de las órdenes de producción, minimizando tiempos de respuesta a la persona encargada de tomar la decisión de la asignación de la producción.

\section{Palabras Clave:}

Órdenes de producción, Optimización, Algoritmo genético, Sistema de producción, Algoritmo evolutivo

\section{Introducción}

En casi todas las empresas los procesos de planeación, programación y control de órdenes de producción son complejos y difíciles de ejecutar, en especial debido a la

\footnotetext{
a Dr. Víctor Quezada-Aguilar, Dr. José Carlos Quezada-Quezada, Dra. Gaby Yolanda Vega-Cano, Universidad Autónoma del Estado de Hidalgo - Escuela Superior de Tizayuca, https://orcid.org/0000-0001-8806-7880, Email: victor_quezad@uaeh.edu.mx; https://orcid.org/0000-0003-3125-3708, Email: jcarlos@uaeh.edu.mx; https://orcid.org/0000-0003-1440-1617, gaby@uaeh.edu.mx ${ }^{\mathrm{b}}$ Dr. Francisco Quiroz-Aguilar, Mtro. Leopoldo Viveros-Rosas, Mtro. Ricardo Rodríguez-Figueroa, Tecnológico de Estudios Superiores de Coacalco, https://orcid.org/0000-0001-8801-6872, francisco_quiroz@tesco.edu.mx; https://orcid.org/0000-0003-2669-906X,
} leopoldo@tesco.edu.mx; https://orcid.org/0000-0003-2213-9401, ricardo_rodriguez@tesco.edu.mx 
gran cantidad de información y el número de variables necesarias para tomar decisiones acertadas al respecto. Uno de los problemas de mayor importancia se presenta en la programación de órdenes de producción, donde la programación de forma adecuada de un conjunto de trabajos que esperan ser procesados por una 0 un conjunto de máquinas dispuestas en correcta secuencia tecnológica, que permita reducir tiempos de ejecución, procesamiento y de entrega, minimizando costos de producción, [1].

Tradicionalmente la planeación y la programación de la producción de los procesos de fabricación se implementa como dos funciones relevantes para la toma de decisiones en la elaboración de productos. La planificación de procesos, es responsable de seleccionar la secuencia de los procesos de fabricación de acuerdo con las especificaciones de diseño del producto, y la programación, de asignar los recursos de fabricación, tales como máquinas y herramientas a los procesos, sujetos a limitaciones tales como el tiempo de liberación, fechas de vencimiento, y la carga de cada máquina.

La toma de decisiones en la planeación de la producción involucra una amplia variedad de temas, incluyendo el programa maestro de producción, [2] , [3], planeación de requerimientos de materiales (MRP), [4] , [5], software de gestión integrada (ERP), [6], [7] y la planeación agregada, [8], [9]. En la actualidad existen investigaciones de planeación y programación de la producción considerando diferentes factores y restricciones donde destacan: Los autores [10], abordaron el problema de planeación y programación de la producción en un entorno de producción multiproducto y multi-proceso considerando el tamaño de lote.

Los autores [11], proponen un sistema que apoya la toma de decisiones en la planeación y programación de la producción a corto plazo en plantas de producción caracterizadas por un proceso de fabricación de una sola operación. išpes Los autores [12], [13] y [14], investigaron el problema de planeación de la producción para múltiples sitios donde consideran a cada sitio como una unidad de producción independiente y paralela, usualmente pertenecen a problemas de planeación agregada. Sin embargo, pocos estudios se han centrado en la liberación y programación de órdenes de producción entre diferentes sitios en la etapa de planeación de la producción hasta el momento. Los autores [1], describen la asignación de lotes de producción en un sistema productivo multi-línea optimizado por la heurística PSO.

En la práctica la secuencia de los procesos son generados istepien la etapa de planificación de procesos, la mejor secuencia se selecciona como el plan de trabajo para servir como entrada en la programación de la producción. Este enfoque es rígido y limita las alternativas que se pueden utilizar en la etapa de programación para la mejora del rendimiento global. Por otra parte, la planificación de tareas que está en un plan de proceso puede causar carga de trabajo de forma desequilibrada, dando lugar a cuellos de botella superfluos. Peor aún, las interrupciones y los cambios en la planeación integrada y de procesos, cerraría la brecha entre la planeación de procesos y programación para mejorar el desempeño de ambas funciones y hacer que el sistema de fabricación sea más sensible a los ambientes dinámicos internos y externos, [15].

La complejidad de la planeación y programación de órdenes de producción dependerá de las características y variables de cada sistema de fabricación, un correcto manejo y control de las variables permitirá que la organización sea competitiva.

El sistema productivo que se analiza en el documento es un sistema lineal considerado básico, sin embargo esquematiza y explica la importancia de una correcta asignación de órdenes de producción. Actualmente la mayoría de las pequeñas y medianas empresas realizan su asignación de producción basándose en la experiencia o intuición la cual no siempre es correcta o idónea para alcanzar la competitividad.

\section{Sistema de Producción}

El sistema de producción tiene 5 estaciones de trabajo con procesamiento consecutivo en una línea productiva, es decir todos los productos pasan por cada una de las estaciones, donde no necesariamente se realizan las mismas operaciones a cada orden de producción. La Figura 1, muestra las estaciones de trabajo.

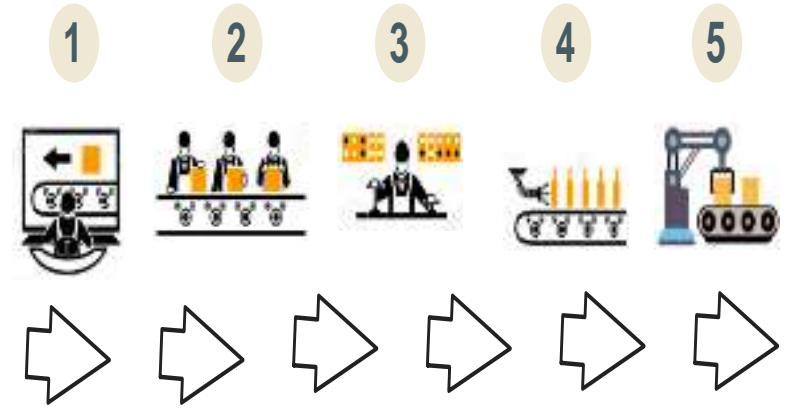

Figura 1. Sistema de procesamiento lineal

La asignación de órdenes de producción al sistema productivo se realiza considerando 3 diferentes programaciones de órdenes de producción 6,10 y 13 
órdenes, respectivamente. Las órdenes de producción se muestran en la tablas 1 .

Tabla 1. Órdenes de producción (6).

\begin{tabular}{|c|c|c|c|c|c|}
\hline \multicolumn{5}{|c|}{ Tiempo de procesamiento en horas-hombre } \\
\hline \multirow{2}{*}{$\begin{array}{c}\text { Órdenes de } \\
\text { producción }\end{array}$} & \multicolumn{5}{|c|}{ Estaciones de trabajo } \\
\cline { 2 - 6 } & $\mathrm{A}$ & $\mathrm{B}$ & $\mathrm{C}$ & $\mathrm{D}$ & $\mathrm{E}$ \\
\hline 1 & 24 & 16 & 15 & 50 & 60 \\
\hline 2 & 30 & 40 & 18 & 40 & 70 \\
\hline 3 & 15 & 22 & 9 & 45 & 65 \\
\hline 4 & 50 & 30 & 7 & 50 & 40 \\
\hline 5 & 20 & 19 & 21 & 25 & 50 \\
\hline 6 & 18 & 17.5 & 32 & 30 & 75 \\
\hline
\end{tabular}

Tabla 2. Órdenes de producción (10).

\begin{tabular}{|c|c|c|c|c|c|}
\hline \multicolumn{5}{|c|}{ Tiempo de procesamiento en horas-hombre } \\
\hline \multirow{2}{*}{$\begin{array}{c}\text { Órdenes de } \\
\text { producción }\end{array}$} & \multicolumn{5}{|c|}{ Estaciones de trabajo } \\
\cline { 2 - 6 } & $\mathrm{A}$ & $\mathrm{B}$ & $\mathrm{C}$ & $\mathrm{D}$ & $\mathrm{E}$ \\
\hline 1 & 28 & 20 & 19 & 60 & 70 \\
\hline 2 & 32 & 42 & 23 & 47 & 77 \\
\hline 3 & 19 & 25 & 13 & 55 & 55 \\
\hline 4 & 40 & 33 & 11 & 55 & 50 \\
\hline 5 & 27 & 21 & 24 & 35 & 55 \\
\hline 6 & 23 & 17 & 15 & 47 & 51 \\
\hline 7 & 31 & 39 & 42 & 58 & 85 \\
\hline 8 & 54 & 65 & 45 & 69 & 89 \\
\hline 9 & 10 & 15 & 20 & 25 & 30 \\
\hline 10 & 9 & 19 & 35 & 39 & 65 \\
\hline
\end{tabular}

Tabla 3. Órdenes de producción (13)

\begin{tabular}{|c|c|c|c|c|c|}
\hline \multicolumn{5}{|c|}{ Tiempo de procesamiento en horas-hombre } \\
\hline \multirow{2}{*}{$\begin{array}{c}\text { Órdenes de } \\
\text { producción }\end{array}$} & \multicolumn{5}{|c|}{ Estaciones de trabajo } \\
\cline { 2 - 6 } & $\mathrm{A}$ & $\mathrm{B}$ & $\mathrm{C}$ & $\mathrm{D}$ & $\mathrm{E}$ \\
\hline 1 & 18 & 30 & 29 & 70 & 80 \\
\hline 2 & 26 & 52 & 33 & 46 & 75 \\
\hline 3 & 21 & 29 & 18 & 65 & 55 \\
\hline 4 & 43 & 23 & 21 & 45 & 52 \\
\hline 5 & 29 & 31 & 44 & 45 & 65 \\
\hline 6 & 26 & 27 & 25 & 47 & 59 \\
\hline 7 & 35 & 49 & 52 & 68 & 75 \\
\hline 8 & 4 & 13 & 28 & 42 & 60 \\
\hline 9 & 5 & 12 & 26 & 36 & 50 \\
\hline 10 & 6 & 11 & 24 & 34 & 40 \\
\hline 11 & 52 & 75 & 55 & 79 & 99 \\
\hline 12 & 14 & 25 & 30 & 45 & 50 \\
\hline 13 & 7 & 15 & 21 & 29 & 35 \\
\hline
\end{tabular}

El proceso de fabricación consiste en mandar a procesar cada orden de producción de forma secuencial, de la orden 1 hasta la última orden. La Figura 2 muestra a dos de las múltiples secuencias de procesamiento.
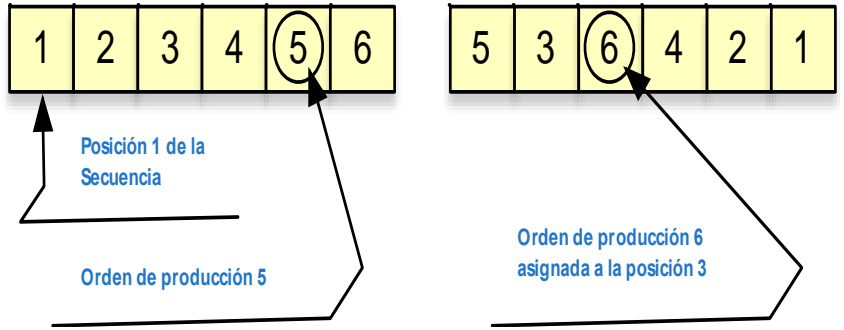

Figura 2. Secuencia de procesamiento.

La secuencia de procesamiento se considera de izquierda a derecha y el número dentro del recuadro hace referencia a la orden de producción. Las combinaciones posibles de asignación se obtienen determinando el factorial del número de órdenes de producción. La tabla 1 tiene 6 ordenes donde el factorial de 6 da como resultado 720 combinaciones posibles.

El número de operarios por estación de trabajo se muestra en la tabla 4.

Tabla 4. Operarios por estación de trabajo

\begin{tabular}{|c|c|c|c|c|}
\hline \multicolumn{5}{|c|}{ Número de operarios por estación de trabajo } \\
\hline $\begin{array}{c}\text { Estación } \\
\text { A }\end{array}$ & $\begin{array}{c}\text { Estación } \\
\text { B }\end{array}$ & $\begin{array}{c}\text { Estación } \\
\text { C }\end{array}$ & $\begin{array}{c}\text { Estación } \\
\text { D }\end{array}$ & $\begin{array}{c}\text { Estación } \\
\text { E }\end{array}$ \\
\hline 3 & 2 & 2 & 3 & 4 \\
\hline
\end{tabular}

Restricciones del sistema de producción

1. El orden de la asignación de procesamiento es de izquierda a derecha en un vector renglón con tamaño igual al número de ordenes de producción.

2. El vector renglón debe contener el número de orden de producción asignado y el total de órdenes de producción.

3. Las estaciones de trabajo sólo puede procesar una orden de producción a la vez.

4. No existen averías ni paros por mantenimiento en las estaciones de trabajo.

5. No existen faltantes de materia prima.

6. La jornada laboral es de 8 horas por día.

Tiempo total de procesamiento del sistema de producción.

Para obtener el tiempo total de procesamiento se considera lo siguiente:

Tiempo de procesamiento: El tiempo de procesamiento en cada estación de trabajo se determina para cada orden de producción.

$$
T P_{i}=\frac{O P_{i}}{\left(O_{i}\right)(T J)}
$$


Donde:

$i=$ Estaciones de trabajo

$T P_{i}=$ Tiempo de procesamiento de la estación de trabajo i.

$O P_{i}=$ Orden de producción de la estación de trabajo i

$O_{i}=$ Número de operarios en la estación de trabajo i

$T J=$ Tiempo de la jornada de trabajo

Tiempo de procesamiento de la orden asignada a la posición 1. Se considera orden 1 a la orden asignada en la posición 1 del vector renglón y se continua con la numeración de las órdenes de acuerdo a la posición que tengan en el vector.

Tiempo de inicio: Para la orden de producción 1, el tiempo de inició es igual a cero para cualquier otra orden de producción el tiempo de inicio es igual al tiempo de procesamiento de la estación de trabajo anterior.

Donde:

$$
\begin{aligned}
& T i=0 ; \text { si } N=1 \\
& T i=T P_{N-1,1} ; \text { si } N \neq 1
\end{aligned}
$$

$N=$ Órdenes de producción

$T i=$ Tiempo de inicio

$T P_{N-1,1}=$ Tiempo de procesamiento de la orden $\mathrm{N}$-1 en la estación de trabajo 1

Tiempo final de procesamiento de la orden 1: El tiempo final se obtiene sumando el tiempo de inicio más el tiempo de procesamiento de cada una de las estaciones de trabajo.

$$
T f_{1}=T i+\sum_{i}^{n} T P
$$

Donde:

$T f_{1}=$ Tiempo final de procesamiento de la orden 1

Tiempo total de procesamiento: Se considera tiempo total de procesamiento al tiempo máximo obtenido en la última estación de trabajo, esté tiempo se obtiene sumando el tiempo de inicio, el tiempo de espera para ser procesado y el tiempo de procesamiento.

$$
\begin{aligned}
& T E_{N, i}=0 ; \text { si } T P_{N, i-1}>T P_{N-1, i-1} \\
& T E_{N, i}=T P_{N-1, i-1}-T P_{N, i-1} ; \text { si } T P_{N, i-1}<T P_{N-1, i-1} \\
& T f_{N}=T i+\sum_{1,1}^{N, i}(T E+T p) \\
& T T_{\text {Sistema }}=\operatorname{Max}\left(T f_{N}\right)
\end{aligned}
$$

Donde:

$T E_{N, i}=$ Tiempo de espera de la orden N, en la estación de trabajo i

$T f_{N}=$ Tiempo final de las órdenes de producción

$T T_{\text {Sistema }}=$ Tiempo total de procesamiento de todas las órdenes de producción

Función objetivo: La función objetivo minimiza el tiempo total de procesamiento.

$$
\operatorname{Min} Z_{1}=T T_{\text {Sistema }}
$$

\section{Algoritmo genético}

Actualmente existen diferentes heurísticas y algoritmos evolutivos que ayudarían a encontrar la solución óptima al problema planteado. Se considera el algoritmo genético por las características del problema, por la facilidad de aplicación y por que es un método sumamente probado en diferentes problemáticas de diferentes ciencias.

Los algoritmos genéticos (AGs) son parte de los algoritmos evolutivos, de ahí que son algoritmos de optimización numérica inspirados en la selección natural y la genética natural. Este algoritmo fue inventado por John Holland en la década de 1960, publicado en su libro de 1975, La adaptación de los sistemas naturales y artificiales, [16].

La solución obtenida por los AEs no garantiza una solución exacta al problema abordado, sino una aproximación donde la calidad de la solución dependerá de los recursos utilizados en la búsqueda de la misma.

Los algoritmos evolutivos, como se mencionó antes, están basados en la teoría de la evolución de Charles Darwin, puntualizando que la evolución de las especies está en relación al principio de selección natural, que favorece la supervivencia y multiplicación de aquellas especies que están mejor adaptadas a las condiciones de su entorno. Otro elemento que Darwin destaca como relevante para la evolución es la mutación, donde los genes son modificados con el objeto de incrementar la calidad de los mismos.

Los AEs responden a un esquema básico común de acuerdo a las siguientes propiedades, [17]:

1. Son procesados todos los individuos obteniendo

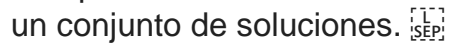

2. La composición de la población se modifica a lo largo de las iteraciones del algoritmo que se denominan generaciones. ilsep?

3. Cada generación incluye un proceso de selección, que da mayor probabilidad de permanecer en la población y participar en las operaciones de reproducción. Los mejores individuos son aquellos que obtienen los mejores valores de la función de adaptación del algoritmo. [s-pepi]

El $A G$ requiere de una serie de parámetros de funcionamiento, como es el tamaño de la población, una vez definida esta, se obtiene la población de individuos de forma aleatoria, los cuales son candidatos a ser la solución del problema en cuestión. En los AGs, los individuos la mayoría de las veces suelen ser cadenas binarias y las llamamos cromosomas, la evolución de la población dependerá de la calidad de los individuos, ya que compiten por aumentar su presencia en la población y participar en las operaciones de reproducción, la 
calidad de los individuos se obtiene por la adecuación o adaptación de cada individuo al ser solución al problema, [17]. La Figura 3 representa un cromosoma de la asignación de ordenes de producción.

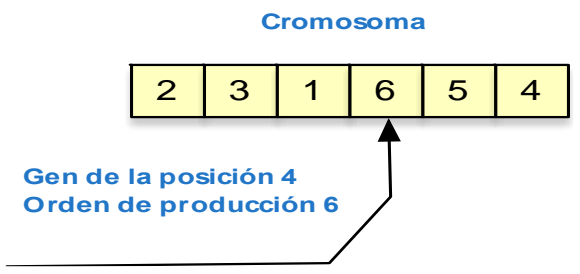

Figura 3. Cromosoma de 6 genes.

Los AGs, así como todos los AEs requieren de especificaciones de paro, es decir cuando el algoritmo dejará de evolucionar y en consecuencia obtener la mejor solución. Por lo general la condición de paro o de terminación es cuando se alcanza un número determinado de generaciones, y este está determinado por el decisor. Otra forma de terminar la evolución se presenta cuando se alcanza una solución de una determinada calidad, [17].

La selección de individuos es de suma importancia y se deben obtener los más capacitados para que éstos sean los que se reproduzcan con más probabilidad de acuerdo con la teoría de Darwin, en la cual los más capacitados son los que deben sobrevivir y crear una nueva descendencia más facultada. El método de selección que se utilizará en la optimización del sistema de producción es el método de selección por ruleta consiste hacer que cada cromosoma tenga una parte de esa ruleta mayor o menor en función a la puntuación que tenga cada uno. Se hace girar la ruleta y se selecciona el cromosoma en el que se pare la ruleta. Obviamente el cromosoma con mayor puntuación saldrá con mayor probabilidad, como se muestra en la Figura 4.

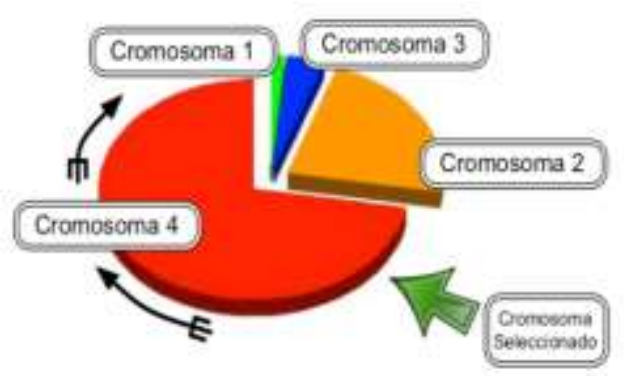

Figura 4. Método de selección por ruleta.

Una vez evaluados los cromosomas y determinado el método de se lección, pasamos al cruce o reproducción. En esta parte los cromosomas realizan intercambio de información en forma similar a la utilizada por un organismo natural se someten a reproducción sexual, la cual se presenta por diferentes métodos. El método que se utiliza en el proceso de optimización lo propone, [18]. Considera una cadena bits de la misma longitud que los cromosomas donde los valores igual a 1 intercambien posiciones para formar al hijo 1 y en los valores igual a 0 intercambian posiciones para formar al hijo 2 como se muestra en la Figura 4.

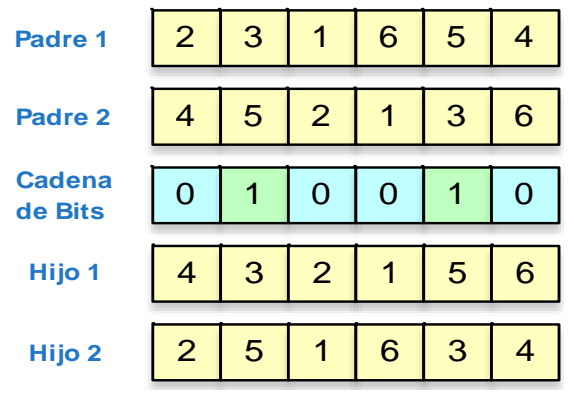

Figura 4. Reproducción de cromosomas

La mutación es utilizada para cambiar aleatoriamente el valor de los genes de forma individual dentro de cada cromosoma. La mayoría de las veces la mutación es utilizada con una probabilidad muy pequeña, rara vez mayor al 1\%. La Figura 5 muestra la mutación del cromosoma.

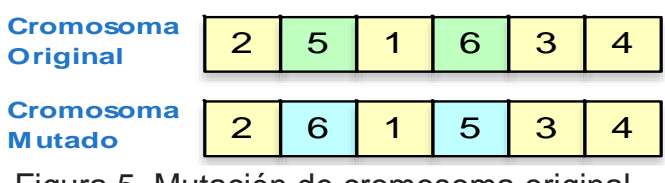

Figura 5. Mutación de cromosoma original.

El algoritmo genético de forma general se representa en la Figura 6. 


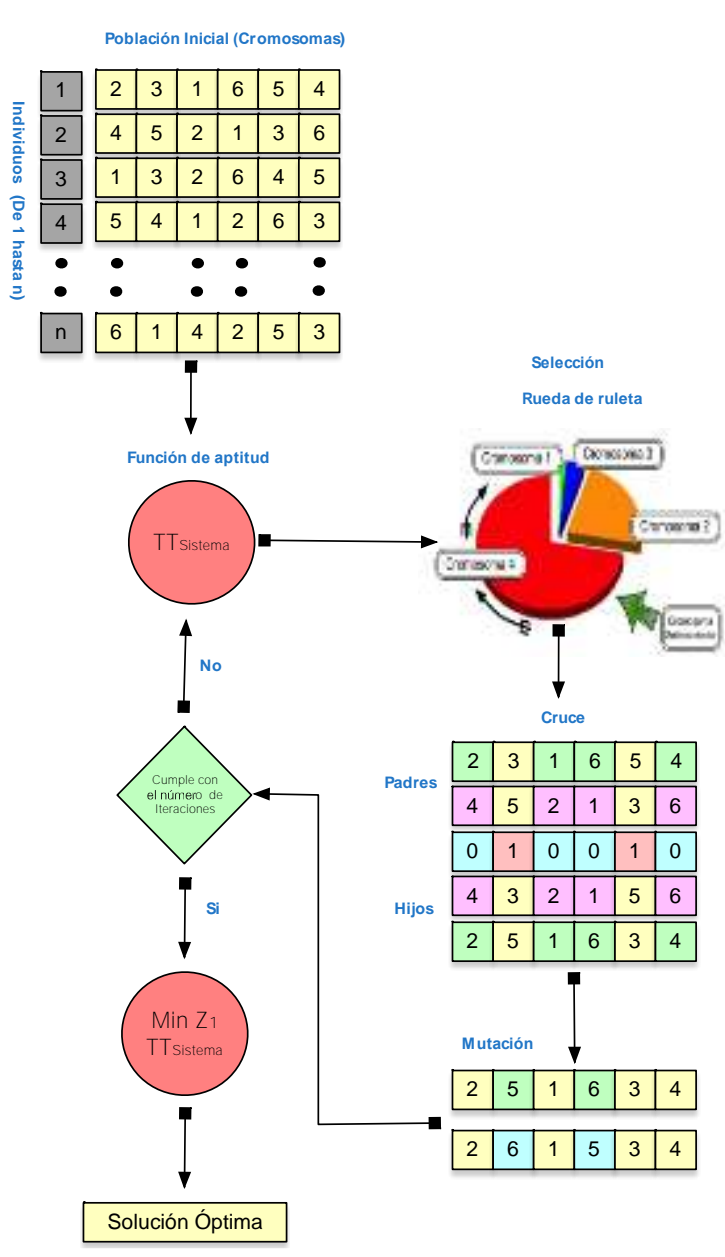

Figura 6. Representación del algoritmo genético.

\section{Resultados}

El simulador del sistema de producción evalúa y optimiza la función objetivo para 6 órdenes de producción con una población inicial de 100 individuos o cromosomas realizando 10 iteraciones, Para 10 órdenes de producción se evalúa con una población inicial de 200 individuos realizando 20 iteraciones. Para 13 órdenes de producción se considera una población inicial de 500 individuos y 50 iteraciones. Los tres problemas de optimización utilizan $1 \%$ de mutación y $60 \%$ de probabilidad de cruce. Los cromosomas 0 soluciones al valor óptimo de la asignación de 6 órdenes de producción se muestran en la tabla 5.

Tabla 5. Soluciones de asignación al problema 1

\begin{tabular}{|c|c|c|c|c|c|c|c|}
\hline № & \multicolumn{6}{|c|}{ Vector de asignación } & $\begin{array}{c}\text { Valor } \\
\text { óptimo }\end{array}$ \\
\hline 1 & 3 & 5 & 6 & 2 & 4 & 1 & \multirow{9}{*}{$\begin{array}{c}15.6875 \\
\text { Días }\end{array}$} \\
\hline 2 & 3 & 5 & 1 & 6 & 2 & 4 & \\
\hline 3 & 3 & 5 & 1 & 4 & 6 & 2 & \\
\hline 4 & 3 & 6 & 2 & 1 & 5 & 4 & \\
\hline 5 & 3 & 1 & 5 & 2 & 4 & 6 & \\
\hline 6 & 3 & 6 & 5 & 2 & 1 & 4 & \\
\hline 7 & 3 & 6 & 1 & 2 & 5 & 4 & \\
\hline 8 & 3 & 5 & 1 & 2 & 6 & 4 & \\
\hline 9 & 3 & 6 & 1 & 4 & 5 & 2 & \\
\hline
\end{tabular}

\begin{tabular}{|l|llllll|}
\hline 10 & 3 & 6 & 2 & 4 & 5 & 1 \\
\hline 11 & 3 & 6 & 5 & 1 & 4 & 2 \\
\hline 12 & 3 & 6 & 2 & 5 & 4 & 1 \\
\hline 13 & 3 & 6 & 5 & 2 & 4 & 1 \\
\hline 14 & 3 & 6 & 4 & 5 & 1 & 2 \\
\hline 15 & 3 & 6 & 2 & 5 & 1 & 4 \\
\hline 16 & 3 & 5 & 6 & 1 & 2 & 4 \\
\hline 17 & 3 & 6 & 1 & 5 & 2 & 4 \\
\hline 18 & 3 & 5 & 1 & 2 & 4 & 6 \\
\hline 19 & 3 & 5 & 6 & 2 & 1 & 4 \\
\hline 20 & 3 & 1 & 6 & 4 & 2 & 5 \\
\end{tabular}

El sistema de producción no se considera robusto en sus operaciones y el número de órdenes de producción en el problema 1 nos lleva a que la optimización tiene como mínimo 20 soluciones para el valor óptimo.

Tabla 6. Soluciones de asignación al problema 2

\begin{tabular}{|c|c|c|c|c|c|c|c|c|c|c|}
\hline № & \multicolumn{9}{|c|}{ Vector asignación } & $\begin{array}{l}\text { Valor } \\
\text { óptimo }\end{array}$ \\
\hline 1 & 10 & 5 & 63 & 1 & 7 & 8 & 9 & 2 & 4 & \multirow{6}{*}{$\begin{array}{c}26.6250 \\
\text { Días }\end{array}$} \\
\hline 2 & 10 & 3 & 65 & 7 & 1 & 8 & 2 & 4 & 9 & \\
\hline 3 & 10 & 6 & 13 & 5 & 7 & 8 & 4 & 2 & 9 & \\
\hline 4 & 10 & 5 & 13 & 6 & 7 & 8 & 4 & 2 & 9 & \\
\hline 5 & 10 & 3 & $\begin{array}{ll}67 \\
6\end{array}$ & 1 & 5 & 8 & 2 & 4 & 9 & \\
\hline 6 & 10 & 1 & 56 & 3 & 7 & 8 & 4 & 2 & 9 & \\
\hline
\end{tabular}

El número de combinaciones posibles considerando 10 órdenes de producción es 5000 veces el número de combinaciones de 6 órdenes, lo que hace al problema de asignación complejo, se puede apreciar en la tabla 6 el número de soluciones para el problema 2 es considerablemente menor comparado con el problema 1.

La tabla 7 muestra la solución óptima considerando 13 órdenes de producción lo que limita a sólo una solución. El problema 3 es 1700 veces el número de combinaciones de 10 órdenes de producción.

Tabla 7. Soluciones de asignación al problema 3

\begin{tabular}{|c|c|c|}
\hline № & Vector asignación & $\begin{array}{c}\text { Valor } \\
\text { óptimo }\end{array}$ \\
\hline 1 & 10915126811723413 & $\begin{array}{c}33.1979 \\
\text { Días }\end{array}$ \\
\hline
\end{tabular}

\section{Conclusiones}

El problema de planeación y asignación de órdenes de producción tiene un grado de complejidad bastante importante, el aumento de las operaciones en el sistema de producción o el aumento de órdenes de producción o el aumento de ambas incrementa la complejidad de tomar una decisión factible a la hora de asignar las órdenes de producción. Aún en la actualidad se sigue tomando está decisión basado en la experiencia o intuición del personal encargado de la planeación, programación y control de la producción. 
El sistema de producción que se propone en el artículo no tiene complejidad o restricciones que lo hagan complejo por lo tanto cuando se tiene un número de órdenes de producción relativamente pequeño como se plantea en el problema 1, el número de soluciones o de asignación de las órdenes de producción es bastante amplio como mínimo se obtuvieron 20 soluciones posibles al valor óptimo. Se podría pensar que incrementando el número de órdenes de producción habría un número de soluciones mayor debido a que se incrementa el número de combinaciones, sin embargo. La simulación del sistema da como resultado la complejidad de tomar una decisión correcta ya que el número de soluciones factibles se va acotando, el problema 2 se redujo a 6 soluciones para el óptimo y el problema 3 a sólo 1 solución.

Actualmente este tipo de problemas se pueden resolver en tiempos muy cortos gracias al avance tecnológico. La simulación del sistema de producción con el problema 1 , 2 y 3 tardo 1,5 y 20 segundos respectivamente en obtener la solución óptima considerando una PC con procesador i5 y 4 MB de RAM, dando tiempos de respuesta al decisor relativamente instantáneos.

Como trabajos futuros se puede considerar evaluar al sistema de producción con algoritmos evolutivos de nueva generación o incrementar la complejidad del sistema de producción acercándolo aun sistema de producción real.

\section{Referencias}

[1] Victor Quezada-Aguilar, Juan Carlos Seck Tuoh-Mora, José Carlos Quezada-Quezada, and Arturo Cuatepotzo-Bravo, "Sistema de producción multi-línea optimizado por PSO," Ingeniería Investigación y Tecnología, vol. XXI, no. 1, pp. 1-11, Enero 2020

[2] Sahin Funda, Robinson E. Powell, and Gao Li Lian, "Master production scheduling policy and rolling schedules in a twostage make-to- order supply chain. ," International Journal of Production Economics, vol. 115, no. 2, pp. 528-541, 2008.

[3] Ray Venkataraman and Jay Nathan, "Master production scheduling for a process industry environment: A case study ," International Journal of Operations \& Production Management, vol. 14, no. 10, pp. 44-53, 1994

[4] Alexandre Dolgui and Caroline Prodhon, "Supply planning under uncertainties in mrp environments ," Annual Reviews in Control, vol. 31 , no. 2 , pp. 269-279, 2007

[5] Vu Thanh Le and Bruce M Gunn , "Sacid Nahavandi. Mrpproduction planning in agile manufacturing ," In Intelligent Systems, 2004. Pro- ceedings. 2004 2nd International IEEE Conference, vol. 2, no. 405-410, 2004.

[6] Ike C. Ehie and Mogens Madsen, "Identifying critical issues in enterprise resource planning (ERP) implementation ," Computers in industry, vol. 56, no. 6, pp. 545-557, 2005.
[8] Abouzar Jamalnia and Mohammad Ali Soukhakian, "A hybrid fuzzy goal programming approach with different goal priorities to aggregate production planning ," Computers \& Industrial Engineering , vol. 56, no. 4, pp. 1474-1486, 2009.

[7] Avi Parush, Adi Hod, and Avy Shtub, "Impact of visualization type and contextual factors on performance with enterprise resource planning systems ," Computers \& Industrial Engineering, vol. 52, no. 1, pp. 133-142, 2007

[9] William B. Lee, Earle Steinberg, and Basheer M. Khumawala, "Aggregate versus disaggregate production planning: A simulated experiment using $\mathrm{ldr}$ and mrp ," The International Journal of Production Research, vol. 21, no. 6, pp. 797-811, 1983.

[10] KF Man, KS Tang, S Kwong, and WH Ip, "Genetic algorithm to production planning and scheduling problems for manufacturing systems ," Production Planning \& Control, vol. 11, no. 5, pp. 443-458, 2000.

[11] J. Józefowska and A. Zimniak , "Optimization tool for shortterm production planning and scheduling," International Journal of Production Economics , vol. 112, no. 1, pp. 109-120, 2008.

[12] Alain Guinet, "Multi-site planning: A transshipment problem," International Journal of production economics , vol. 74, no. 1 , pp. 21-32, 2001

[13] Stephen CH Leung, Sally OS Tsang, Wan Lung Ng, and Yue Wu, "A robust optimization model for multi-site production planning problem in an uncertain environment," European journal of operational research, vol. 181, no. 1, pp. 224-238, 2007.

[14] Christian H Timpe and Josef Kallrath , "Optimal planning in large multi-site production networks ," European Journal of Operational Research, vol. 126, no. 2, pp. 422-435, 2000.

[15] Luping Zhang and TN Wong , "Solving integrated process planning and scheduling problem with constructive meta-heuristics ," Information Sciences, vol. 340, pp. 1-16, 2016.

[16] David A Coley, An introduction to genetic Algorithms for Scientists and Engineers. USA: World Scientific, 2005.

[18] Z.X. Guo , W.K. Wong, Zhi Li , and Peiyu Ren, "Modeling and Pareto optimization of multi-objective order scheduling problems in production planning," Computers \& Industrial Engineering , vol. 64, pp. 972-986, 2013.

[17] Lourdes Araujo and Carlos Cervigón , Algoritmos evolutivos: un enfoque práctico. México, México: Alfaomega, 2009, vol. 1.

[19] P. Sawyer, "Planning and controlling production with mrp II ," Chemical Engineer, pp. 32-41, 1990.

[20] Md Abdul Wazed, Shamsuddin Ahmed, and Yusoff Nukman , "A review of manufacturing resources planning models under different uncertain- ties: State-of-the-art and future directions ," South African Journal of Industrial Engineering , vol. 21, no. 1, pp. 17-34, 2010. 